\title{
Problemas que dificultan una mejor utilización de la Didáctica de las Ciencias en la Formación del Profesorado y en la Enseñanza Secundaria
}

\author{
Problems that hinder a better use of Science Education in the teacher's training and \\ Secondary Education
}

\section{Problemas que impedem um melhor uso do ensino de ciências na for-mação de professores e no ensino secundário}

Jaime Carrascosa-Alís ${ }^{1}$

M. Consuelo Domínguez-Sales

Recibido: mayo de 2017

Aceptado: agosto de 2017

Para citar este artículo: Carrascosa-Alís, J., y Domínguez-Sales, C. (2017). Problemas que dificultan una mejor utilización de la Didáctica de las Ciencias en la Formación del Profesorado y en la Enseñanza Secundaria. Revista Científica, 30 (3), 167-180. Doi: https://doi.org/10.14483/23448350.12289

\section{Resumen}

La didáctica de las ciencias experimentales se puede considerar como un cuerpo global y coherente de conocimientos específicos entre los que existe un "núcleo duro" donde se hallan, entre otros, aqueIlos contenidos más directamente relacionados con aspectos claves para la enseñanza y el aprendizaje de las ciencias. Su apropiación, por parte del profesorado, es necesaria para poder avanzar hacia una enseñanza de las ciencias de mayor calidad. Sin embargo, dicha apropiación exige la superación de una serie de problemas que deben ser tenidos muy en cuenta. En este trabajo se analizan algunos de ellos y se justifican algunas propuestas para poder avanzar en su solución.

Palabras clave: didáctica de las ciencias experimentales; formación del profesorado de ciencias; enseñanza de didáctica de las ciencias experimentales; aprendizaje de didáctica de las ciencias experimentales.

\begin{abstract}
Science Education today can be considered as a whole and coherent body of specific knowledge with a "hard core" in which the most directly issues related to key aspects of teaching and learning can be found. The appropriation of this knowledge by teachers is required in order to move towards a higher quality teaching in the field of the experimental sciences. However, this appropriation is not an easy task, and requires overcoming a number of problems which must be taken into account. In this paper, some of these problems are discussed, and some proposals to make progress in solving them are justified.
\end{abstract}

Keywords: science education; science teacher's training; teaching science education; learning science education. 


\section{Resumo}

A Didática das Ciências Experimentais já pode ser considerada como um corpo global e coerente de conhecimentos específicos, com um "núcleo duro" no qual são encontrados, entre outros, aqueles conteúdos mais diretamente relacionados com aspectos chaves para o ensino e a aprendizagem das ciências. Sua apropriação por parte dos professores é algo necessário para poder avançar em direção a um ensino das ciências de melhor qualidade. No entanto, tal apropriação exige a superação de uma série de problemas que deve ser levada em conta. Neste artigo, alguns desses problemas são analisados e algumas propostas são justificadas para poder avançar em sua solução.

Palavras-chaves: didática das ciências experimentais, formação de professores de ciências, ensino de didática das ciências experimentais, aprendizagem de didática das ciências experimentais.

\section{Introducción}

Afortunadamente, ya quedó atrás esa época en la que era frecuente referirse a las "características" que debía tener un profesor para impartir una buena enseñanza, las cuales solían verse como una especie de cualidades naturales que solo podían mejorarse a partir de la experiencia.

El hecho de que hoy nos refiramos de forma explícita a competencias que deben adquirirse (Cortés et al., 2015), en lugar de aludir a características o cualidades más o menos innatas, supone una diferencia fundamental que rompe con esa visión esencialista anterior y permite pensar en la formación del profesorado y en la renovación de la enseñanza, como el proceso de adquisición y desarrollo de unos conocimientos teórico-prácticos, fruto de investigaciones e innovaciones que continuamente están siendo experimentadas y evaluadas. En el campo de las ciencias experimentales (donde se centra todo este trabajo), dichos conocimientos no están formados por unos cuantos bloques dispersos, sino que se ven estrechamente vinculados entre sí, abarcan muchos de los aspectos que se consideran claves para la enseñanza y aprendizaje y constituyen una parte fundamental de lo que se conoce como didáctica de las ciencias experimentales (en adelante DCE).

Un objetivo básico de la DCE es enseñar a enseñar bien, admitiendo que siempre es posible mejorar el aprendizaje y que podemos elaborar las estrategias más adecuadas para conseguirlo. Ello supone, esencialmente, estudiar los problemas más significativos de la enseñanza y aprendizaje de las ciencias experimentales (Física, Química, Biología, etc.) y elaborar propuestas educativas teóricamente fundamentadas con las que poder avanzar en su solución. Se trata, en definitiva, de generar conocimientos que permitan favorecer continuamente el proceso de enseñanza y aprendizaje de las ciencias o, como han señalado algunos autores, de utilizar la DCE como la ciencia de enseñar ciencias (Sanmartí e Izquierdo, 2001).

Atendiendo al objetivo anterior, se desarrollaron (desde finales de la década de 1970), diversas líneas de investigación sobre determinados aspectos claves para la enseñanza y aprendizaje de las ciencias en el aula, las cuales ya han producido conclusiones y resultados muy interesantes (que podrían entenderse como una parte esencial del núcleo duro de la DCE) y sobre los que existe un amplio consenso. Entre otras: naturaleza de la ciencia y del trabajo científico; ideas alternativas y aprendizaje de conceptos científicos; trabajos prácticos; resolución de problemas de lápiz y papel; relaciones entre ciencia, tecnología, sociedad y medio ambiente; actitudes hacia la ciencia y su aprendizaje; evaluación, etc.

Sin embargo, la apropiación de los contenidos que conforman dicho núcleo duro no es una tarea simple. Por el contrario, exige la superación de una serie de problemas que deben ser tenidos muy en cuenta (especialmente en la formación del profesorado) ya que, pueden actuar como verdaderos obstáculos que dificultan el avance hacia una enseñanza de las ciencias de calidad, susceptible de ser continuamente mejorada. Dicha mejora no solo es un objetivo inherente a la propia DCE sino que, además, es una demanda social, puesta 
de manifiesto por diversos grupos de expertos que han estudiado el problema de la alarmante pérdida de interés del alumnado hacia los estudios científicos y la disminución en el número de estudiantes que los cursan (Solbes, Montserrat y Furió, 2007; Vázquez y Manassero, 2008). En el Ilamado Informe Rocard se insiste reiteradamente en vincular toda esta problemática a la forma en que la ciencia es habitualmente enseñada, por lo que se recomienda explícitamente y de forma reiterada, un mayor apoyo institucional a la formación didáctica del profesorado de ciencias (Rocard, et al., 2007).

Tiene sentido, pues, plantearse cuáles son los problemas más importantes a los que nos enfrentamos desde la DCE permite para mejorar la formación del profesorado de ciencias y con ello el proceso de enseñanza y aprendizaje de las asignaturas de ciencias. En este trabajo, se pretende dar una visión panorámica (aunque no exhaustiva) de algunos de esos problemas y se proponen también algunas medidas para avanzar en su solución.

\section{Un conocimiento inadecuado (escaso, superficial, limitado...) de la materia a enseñar}

Se ha dicho que un profesor que no posee conocimientos específicos de cierta profundidad sobre la materia que enseña es un profesor inseguro y excesivamente dependiente de los libros de texto, lo que se traduce en una merma en la calidad de su enseñanza; pero también es un profesor con serias dificultades para llevar a cabo cualquier pequeña innovación didáctica en sus clases, lo que frena cualquier intento de mejora. Todo ello permite comprender la necesidad de una buena formación científica en la materia a enseñar. Sin embargo, tal y como se constata reiteradamente en diversos cursos de formación, muchos futuros profesores (y también profesores en activo) presentan graves deficiencias en la comprensión y manejo de conceptos y principios básicos que han de enseñar. Incluso se detecta, con cierta frecuencia, la existencia entre ellos de determinadas ideas alternativas bien conocidas y documentadas. Esta falta de conocimientos es un obstáculo importante que no puede ser soslayado, lo que nos deja afirmar que en la formación docente se deben incluir temas que permitan profundizar más, sobre todo, en aquellos contenidos básicos de las disciplinas que se habrán de enseñar. No es admisible, por ejemplo, que de una facultad de educación (sea del lugar que sea) puedan salir futuros profesores de ciencias que apenas conocen (o desconocen por completo) una parte sustancial de los contenidos de Física que deben enseñar.

Por otro lado, es preciso tener en cuenta que algo tan aparentemente claro y homogéneo como conocer la materia a enseñar implica en realidad unos conocimientos profesionales muy diversos. Concretamente, además de conocer leyes, principios, modelos, teorías y demás contenidos conceptuales que forman parte de la materia a enseñar, es preciso también que todos los profesores de ciencias (Gil et al.., 1991):

a. Tengan conocimientos de Historia de la Ciencia, útiles para contribuir a una mejor enseñanza de sus materias y para facilitar una mejor comprensión de la naturaleza de la Ciencia (Aragón-Méndez, et al., 2016). A modo de ejemplo, podemos citar la necesidad de conocer bien los obstáculos (de todo tipo) más importantes que debieron superarse en la elaboración y consolidación de los nuevos conocimientos, lo que constituye una ayuda imprescindible para comprender muchas de las dificultades de los alumnos. Así, un profesor que conozca la vigencia, el alcance y las características fundamentales que tuvieron, por ejemplo, la mecánica aristotélico-escolástica, el calórico o el lamarckismo, estará mucho más receptivo cuando en su clase surjan ideas que relacionen la fuerza con la velocidad, el calor con una sustancia o la longitud de la cola de unos ratones con el uso que de este órgano hubieran hecho sus progenitores, 
al tiempo que dispondrá, también, de mejores elementos de juicio para comprender la persistencia de tales ideas y plantearse su proceso de cambio hacia las que trata de enseñar.

b. Conozcan las estrategias metodológicas empleadas en la construcción de los conocimientos, es decir, la forma en que los científicos abordan los problemas, las características más notables de su actividad, los criterios de aceptación y validación de las teorías científicas, etc. Sin esto, el profesor difícilmente puede plantearse el cambio de ideas espontáneas o simplistas sobre la ciencia y el trabajo científico entre sus alumnos, desarrollar en sus clases estrategias metodológicas realmente coherentes con la investigación científica o, simplemente, asumir el objetivo (explícito en la mayoría de los currículos educativos actuales) de que sus alumnos adquieran y desarrollen la competencia científica.

c. Conozcan las estrechas relaciones existentes entre ciencia-tecnología, sociedad y medio ambiente (en adelante CTSA), asociadas a la construcción de conocimientos. Algo imprescindible cuando se habla de la necesidad de una alfabetización científico-tecnológica de toda la ciudadanía o de mostrar la complejidad que conlleva la toma de determinadas decisiones. Resulta especialmente importante que sepan cómo pueden contribuir desde sus materias a la educación para la sostenibilidad, una tarea absolutamente necesaria para poder avanzar en la solución de los graves problemas (cambio climático, contaminación pluriforme, agotamiento de recursos, etc.) que amenazan al planeta en su conjunto (Vilches y Gil Pérez, 2009).

d. Tengan conocimientos de los desarrollos científicos y tecnológicos recientes, así como de sus perspectivas futuras, tanto para poder transmitir una visión dinámica no cerrada de la ciencia, como para utilizar adecuadamente algunos de estos (TIC) como nuevas herramientas didácticas con un indudable potencial para aumentar la motivación y el rendimiento, posibilitar trabajos prácticos virtuales sin riesgo ni residuos tóxicos, mejorar la comprensión de determinados fenómenos, etc. Evitando en todo caso, que la necesaria renovación de la enseñanza se limite al uso de nuevas tecnologías, pero con las mismas viejas metodologías de siempre (Marchesi et al.., 2005). Es lo que sucede, por ejemplo, si usamos una pizarra digital para enseñar a resolver problemas, pero lo hacemos mediante la simple transmisión verbal ante alumnos pasivos, o cuando utilizamos computadoras y sofisticados sensores en prácticas de laboratorio, pero las seguimos planteando como simples recetas de cocina, etc.

\section{El pensamiento docente espontáneo}

Otro problema importante es aquello que el profesor sabe sin ser muchas veces consciente de que lo sabe (Gil et al., 1991), ya que a veces se trata de concepciones simplistas sobre la enseñanza y el aprendizaje, originadas por determinadas vivencias que muchos profesores han tenido durante su larga etapa como alumnos. El carácter reiterado de esas vivencias hace que dichas concepciones lleguen a establecerse como evidencias de "sentido común" que, por tanto, no hace falta cuestionarlas. Además, es preciso tener en cuenta que esa impregnación ambiental dificulta también su transformación, de forma análoga a como ocurre en los alumnos con algunas ideas previas respecto a determinados conceptos científicos (Carrascosa, 2005). Algunos ejemplos de este tipo de pensamiento son:

a. La idea de que enseñar es una tarea fácil en la que normalmente basta con conocer los contenidos (principios, modelos, teorías...) de la materia (De Pro, 2009), algo de sentido común y un poco de práctica. Esta era una concepción simplista muy extendida hace años (lo que explica, en parte, la deficiente atención real que a menudo se ha prestado a la formación profesional para la docencia) y que todavía no ha sido del todo superada. Algunos autores (Gil-Pérez y 
Vilches, 2004) la utilizan para explicar, al menos en parte, el hecho de que la docencia universitaria sea vivida por bastantes profesores como una carga que quita tiempo para otras tareas investigadoras que se consideran más prioritarias (y productivas), lo que, según estos mismos autores, revela una concepción de la enseñanza como algo estándar y repetitivo, algo que en definitiva "puede hacer cualquiera"; mientras que la investigación, por el contrario, suele verse como una tarea mucho más compleja, abierta y creativa, reservada a los mejores. La existencia de estas grandes diferencias en cuanto a la prioridad de la investigación frente a la docencia favorece también unas clases tradicionales, fundamentalmente expositivas, muy alejadas de la metodología y forma de trabajo que esos mismos profesores utilizan en sus investigaciones (Waldrop, 2015). En cualquier caso, conviene tener en cuenta que esta situación, siempre que se produce, afecta negativamente a la calidad de la enseñanza de cualquier materia (DCE, incluida).

b. La existencia de concepciones simplistas acerca de la Ciencia, el trabajo científico y los propios científicos (empirista y ateórica, descontextualizada, elitista, aproblemática y ahistórica, rígida y algorítmica, etc.). No podemos detallar aquí dichas concepciones, que ya han sido expuestas y comentadas en diversos trabajos de investigación, como, por ejemplo: Fernández et al. (2005), García-Carmona y Acevedo (2016), etc., pero sí conviene insistir en el hecho de que las ideas que el profesor tenga respecto a la naturaleza de la Ciencia, el trabajo científico y los propios científicos pueden influir mucho en su enseñanza, desde el enfoque que dé a los trabajos prácticos, el sistema de evaluación que utilice, los contenidos que seleccione... hasta los métodos de enseñanza que lleve a cabo en sus clases.

c. La idea de que el fracaso escolar y las actitudes negativas de muchos alumnos hacia algunas asignaturas de ciencias, obedecen exclusivamente a causas externas (sobre las que el profesor difícilmente puede actuar), como la influencia de un medio socialmente desfavorecido, problemas familiares, diferencias supuestamente innatas en capacidad intelectual o la complejidad intrínseca atribuida a las materias científicas, es otra concepción simplista (o reduccionista) que conviene considerar. Se trata de una idea de carácter exculpatorio y que se halla bastante extendida entre los educadores. Su existencia dificulta la búsqueda y el estudio de la posible relación del fracaso escolar y desinterés hacia las ciencias, con otros factores inherentes al propio proceso educativo que, sin duda, también pueden influir (y sobre los que el profesorado sí que puede actuar). Entre otros: la metodología empleada para enseñar, los materiales de trabajo utilizados, la forma de evaluar y calificar, el funcionamiento del aula y del centro escolar o la relación entre el profesor y sus alumnos.

d. Ideas y actitudes simplistas sobre la evaluación del aprendizaje del alumnado en materias científicas (véase Alonso, Gil y Martínez Torregrosa, 1992) como, por ejemplo: la supuesta objetividad de las calificaciones obtenidas en las pruebas realizadas, pensar que evaluar consiste en calificar al final lo que el alumno ha aprendido antes, reducir la evaluación a lo más fácilmente medible, escasa coherencia entre los contenidos tratados y los evaluados, etc.

\section{Separación de los contenidos educativos de los científicos}

En la enseñanza de las ciencias tiene gran importancia desarrollar en el alumnado la competencia científica. Ello implica, entre otras cosas, enseñar a plantearse problemas de interés y saber precisarlos, elaborar hipótesis fundadas y posibles diseños para su contrastación, realizar análisis críticos, argumentar, modelizar, búsqueda de coherencia, etc.; rompiendo con hábitos de pensamiento muy enraizados, lo que supone un verdadero cambio 
metodológico. Sin embargo, hay que ser conscientes de que dicho cambio conlleva unos determinados requerimientos y que no es posible avanzar en él dejando de lado la adquisición de unos contenidos conceptuales. En efecto: no se puede enseñar la competencia científica en abstracto, como tampoco es posible llevar a cabo ninguna investigación científica fuera de un marco conceptual determinado. Pues bien, en la enseñanza de la DCE ocurre algo parecido, pudiendo afirmarse que los contenidos educativos van indisolublemente unidos a los científicos, de otra forma queda desvirtuada y no es tal. En otras palabras: no se puede enseñar DCE sin saber ciencias y sin utilizar contenidos propios de las materias científicas en el proceso.

Sin embargo, no es raro encontrar cursos de formación en los que esta se concibe como una simple suma de unos contenidos científicos (esencialmente conceptuales), por un lado, y unos elementos genéricos de educación (esencialmente psicología, pedagogía y sociología) por otro (Michelini et al., 2013). Esta separación de los contenidos educativos de los científicos se ha mostrado muy poco eficaz (Michelini, 2004; Viennot et al., 2005). En efecto, si una determinada estrategia de enseñanza, por ejemplo, se estudia ignorando los contenidos en los que va a ser implementada, los profesores probablemente no sabrán identificar los aspectos esenciales, ni adaptar a su asignatura aquello que les ha sido presentado en términos demasiado abstractos, pudiéndose generar actitudes de rechazo hacia unos planteamientos que no conectan claramente con los problemas percibidos por ellos al tratar de enseñar los contenidos de su materia. En definitiva: no se puede enseñar a enseñar sin tener muy en cuenta el contenido que hay que enseñar. En las facultades de educación, y en la formación del profesorado de ciencias en general, es necesario asegurarse de que se cumpla este principio, porque en ocasiones se observa una clara separación entre quienes imparten los contenidos científicos (con una metodología tradicional que ignora los avances de la DCE) y quienes imparten los contenidos didácticos (con una presencia escasa o nula de contenidos científicos de las materias que los futuros profesores habrán de enseñar).

\section{Falta de materiales educativos innova- dores adecuados}

Existen resultados de gran interés en torno a aspectos claves para la enseñanza y aprendizaje de las ciencias, como los citados con anterioridad a título de ejemplo (ideas alternativas, trabajos prácticos, resolución de problemas de lápiz y papel... o la misma evaluación), que son fruto de numerosas investigaciones ya realizadas y sobre los cuales se da un importante consenso. Sin embargo, la situación es radicalmente diferente en lo que se refiere a la existencia de materiales educativos innovadores, elaborados de forma coherente con las orientaciones didácticas emanadas de esas investigaciones. Dicha elaboración es una tarea necesaria (y, por otra parte, inacabable) para que esos resultados puedan, en principio, traducirse en mejoras realmente significativas en el proceso de enseñanza y aprendizaje de las ciencias.

En efecto, una conclusión ampliamente aceptada entre los investigadores de DCE es la necesidad de impulsar estrategias de indagación e investigación en el alumnado, dentro de lo que es la competencia científica. Esto es lo que se enfatiza, por ejemplo, en un documentado estudio sobre la formación del profesorado de ciencias, realizado recientemente en Estados Unidos (National Academies of Sciencies, Engineering and Medicine, 2015), en el que se afirma: "Research has shown that students best understand scientific ideas when they actively apply their knowledge while engaging in the practices of science, for example, modeling, developing explanations or solutions, and arguing about evidence...".

A ello hemos de añadir que, en nuestra opinión, esta estrategia no puede desarrollarse con éxito si se hace de manera parcelada (por ejemplo, solo en las prácticas de laboratorio), sino que hay 
que extenderla también a otras actividades (como la resolución de problemas de lápiz y papel o la introducción de conceptos). Ahora bien, la DCE no puede (ni debe) limitarse a dar esas recomendaciones, una limitación que de acuerdo con Vilches y Gil-Pérez (2013) podría relacionarse con la existencia de una concepción según la cual las investigaciones deberían centrarse en estudiar problemas relativos a la enseñanza y aprendizaje de las ciencias, pero sin descender a la elaboración de propuestas de actuación concretas en el aula (lo que sería ya calificado como "simple" innovación). Esta situación es coherente, por otra parte, con que en algunas revistas de investigación didáctica se rechacen frecuentemente aquellos trabajos que se consideren de innovación, por muy fundamentados que puedan estar. No se tiene en cuenta, sin embargo, el hecho de que, análogamente a como ocurre en otros campos de la investigación científica como la Física o la Química, tampoco pueden darse realmente por válidos los análisis críticos o las explicaciones a los problemas estudiados en una investigación didáctica hasta que no vayan acompañados de pruebas sólidas que muestren cómo al incidir sobre las supuestas causas, se producen resultados coherentes con las hipótesis manejadas y eso requiere, en muchos casos, de la innovación en el aula.

Es necesario, pues, impulsar desde la propia DCE la elaboración de trabajos prácticos, de colecciones de problemas, de introducciones de conceptos, etc., siguiendo las orientaciones de las investigaciones realizadas en cada caso y, finalmente, el diseño de temas o unidades didácticas completas (incluyendo propuestas para su evaluación), en las que se integren todas estas aportaciones innovadoras (junto con otras) de forma coherente, implicando en ello al profesorado de aula. Eso también debería formar parte de la DCE. Sin embargo, los proyectos de estas características ya realizados y experimentados, impulsados por alguna universidad o institución académica de reconocido prestigio son todavía demasiado escasos y, sobre todo, poco conocidos por la mayoría del profesorado de ciencias. Podemos mencionar, a título de ejemplo el, ya clásico, Children Learning In Science Project, dirigido por Rosalind Driver y elaborado en la Universidad de Leeds, con la colaboración del profesorado de aula al que iba dirigido y que se experimentó (a finales de la década de 1980) como un intento de plasmar las orientaciones de cambio conceptual, en la elaboración de temas completos para ser impartidos a los alumnos. Otros proyectos más actuales son los National Science Education Standards de Estados Unidos o los Pollen y Sinus-Transfer en la Comunidad Europea (citados en Rocard et al., 2007). Señalar también, la existencia de Programas de Actividades (ved ejemplos en Gil et al.., 1991; Carrascosa et al., 2014³) como instrumentos con los cuales los alumnos pueden, con la ayuda y orientaciones que sean necesarias por parte del profesor, construir conocimientos desarrollando la competencia científica.

\section{Una formación didáctica limitada a temas puntuales que se presentan inconexos}

En nuestra opinión, para que la DCE pueda ser aprovechada con éxito por el profesorado, es preciso enseñarla de forma que se pueda percibir la conexión existente entre los distintos temas que forman parte de lo que hemos denominado anteriormente como su núcleo duro. En efecto, una formación puntual en un solo aspecto (por ejemplo, en "introducción de conceptos") en el que se proponen determinados enfoques $u$ orientaciones innovadoras, a pesar de su indudable interés intrínseco, antes o después acabará en nada si no se amplía también a otros aspectos claves (sin que por eso se den incoherencias) y no se refleja todo ello adecuadamente en el proceso evaluador. Solo de esta forma, profesores y profesoras serán capaces de articular todos los aspectos claves a los que

O en la web, véase: didacticafisicaquimica.es 
nos hemos referido (introducción de conceptos, trabajos prácticos, problemas de lápiz y papel, relaciones CTSA, etc.) e integrarlos, junto con otros, de forma funcional en la elaboración de unidades didácticas de Física, Química, Biología, etc.; y también en el propio sistema de evaluación de cada una de dichas unidades, con el propósito de aplicar y experimentar todo ello en el aula. Diversos autores, conscientes de este problema, apuntan la necesidad de una formación intensiva del profesorado en DCE, capaz de superar estas limitaciones (Solbes et al., 2004; Bañas et al., 2009).

Así pues, conviene desde la DCE impulsar toda una serie de medidas que permitan avanzar en la solución a este problema como, por ejemplo: 1) que los contenidos didácticos específicos manejados en la formación del profesorado estén conectados entre sí y se muestre en ellos dicha conexión de forma explícita (Carrascosa, et al., 2014); 2) una elevada coordinación entre los profesores que imparten los contenidos, que habrán de asegurarse también de que sus alumnos perciben toda esa coherencia; 3) cursos de formación docente de larga duración, que permitan la identificación y transformación de posibles concepciones simplistas y en los que se traten la mayor parte posible de los contenidos básicos que conforman el núcleo duro de la DCE, incluyendo un bloque específico sobre cómo se pueden integrar esos contenidos (o sus implicaciones para la enseñanza de las ciencias), en la elaboración de unidades didácticas concretas (Física, Química, etc.) para ser utilizadas con los alumnos en el aula (véase Verdú et al., 2002 o la página web: didacticafisicaquimica.es).

\section{Metodología para el aprendizaje de los con- tenidos de didáctica de las ciencias incohe- rente con la propia didáctica de las ciencias}

Es un hecho constatado, desde hace ya tiempo, que una parte de los cursos de formación en DCE están diseñados para transmitir los resultados de las investigaciones educativas, tomando a los profesores que los reciben como simples receptores-consumidores. Sin embargo, si la enseñanza por transmisión verbal de conocimientos ya elaborados presenta serias deficiencias con el alumnado... ¿por qué habría de funcionar bien en la formación del profesorado? Como afirma Kortaghen (2010): algo importante debe fallar si todavía se sigue ese enfoque tradicional en la formación del profesorado.

Ya se ha comentado anteriormente que, al igual que los alumnos tienen ideas alternativas (a veces muy enraizadas) respecto a determinados conceptos básicos, una gran parte del profesorado en formación tiene también un pensamiento docente de "sentido común" y algunas creencias previas cuyo cambio supone un desafío fundamental (Russell y Martin, 2007). Esta similitud es, sin duda, importante. Pero lo que realmente nos interesa destacar aquí es que dicho desafío, al igual que ocurre en el caso de los alumnos, difícilmente puede afrontarse con éxito mediante un modelo de enseñanza basado en la simple transmisión-recepción verbal de conocimientos. Además, la transmisión verbal tampoco permite que los profesores en formación experimenten en sí mismos muchas propuestas educativas innovadoras, que es, precisamente la forma más eficaz de conocerlas y valorarlas. Resulta absurdo, por ejemplo, hablar de propuestas didácticas y estrategias de enseñanza basadas en una metodología activa y participativa o de cómo desarrollar en los alumnos la competencia científica, mediante simples lecturas, conferencias y evaluaciones tipo test, y más absurdo aún es pretender que luego el profesorado así formado pueda poner todo ello en práctica de forma eficaz.

Por otra parte, conviene insistir en que el uso de nuevas tecnologías de información y comunicación (TIC), a pesar de su indudable interés para la enseñanza en general y de las ciencias en particular, no supone por sí mismo una garantía de que la apropiación de los contenidos tratados se realice con una metodología adecuada (como ya se ha comentado anteriormente). Incluso, puede haber cursos masivos de formación didáctica on line para profesorado de secundaria en los que las 
estrategias de enseñanza se basen exclusivamente en la simple transmisión de conocimientos, aunque en este caso dicha transmisión, y su correspondiente evaluación (generalmente tipo test), se realicen por vía telemática.

En definitiva: para poder desarrollar una formación didáctica de calidad, es preciso que exista una elevada coherencia entre lo que se hace y cómo se hace al enseñar DCE y lo que, durante ese proceso, se dice que el profesorado ha de hacer y cómo lo debe hacer para enseñar ciencias a sus alumnos en un aula.

\section{Fractura existente entre actividad investi- gadora y actividad docente}

La mejora de la enseñanza requiere no solo abordar los problemas desde una óptica investigadora sino, también, que el conocimiento generado sea compartido por el profesorado. A pesar de ello, y aunque esto pueda parecer algo obvio, la realidad es que, en el campo educativo la fractura existente entre investigación y docencia ha sido (y sigue siendo) demasiado grande (Russell y Martin, 2007; Solbes et al., 2013). Muchos profesores no parecen muy proclives a incorporar en sus clases los avances de la investigación educativa (Martín, Prieto y Jiménez, 2015) y miran a los investigadores con recelo, viéndolos en general, como teóricos que desconocen la realidad de las aulas. Existen diversas explicaciones a esas reticencias. A continuación, comentaremos un par de ellas:

En primer lugar, es preciso tener muy en cuenta las condiciones laborales de una gran parte del profesorado, con aulas masificadas, horarios lectivos muy extensos, bajos salarios, etc., (situación que se agrava en la enseñanza privada y especialmente en algunos países donde muchos profesores han de recurrir al pluriempleo para poder vivir dignamente).

En segundo lugar, el que alguien de fuera explique qué hay que hacer (sin que el profesorado al que se dirige haya participado mínimamente en ese proceso), con el convencimiento de que basta exponer nuevas propuestas didácticas con claridad y una buena fundamentación, para que rápidamente sean aceptadas y aplicadas en el aula, es una pretensión, cuando menos, bastante ingenua y que ha sido cuestionada por muchos autores que plantean la necesidad de que los profesores participen en procesos de investigación y de innovación, para favorecer su implicación efectiva en la mejora de la enseñanza (Hewson, 2007; Wallace y Loughran, 2012; Taylor, et al.; 2012). Por ello, al igual que Ritchie (2008), pensamos que la investigación realizada por y con los profesores tienen un efecto transformador muy superior que aquella llevada a cabo en ámbitos académicos para y sobre los profesores.

Esta situación, al menos en lo que se refiere a la DCE, parece estar mejorando algo en los últimos años. Como lo demuestra el gran número de revistas específicas existente, lo que evidencia, no solo una abundancia de investigaciones en este campo, sino también un mercado que las consume y que va en aumento. No obstante, la relación entre el profesorado de ciencias de secundaria y la investigación didáctica es todavía escasa (De Pro, 2009) y se precisa un mayor esfuerzo por aumentarla (Carrascosa et al., 2014). En otras palabras: la investigación didáctica no producirá un impacto real en la docencia, a menos que el profesorado se implique también en ella. Por tanto, es preciso un mayor esfuerzo colectivo para superar todos los obstáculos que dificultan dicha implicación (Oliva, 2011). No solo por parte del propio profesorado afectado, sino también por las autoridades educativas correspondientes, que han de implementar políticas adecuadas y poner a disposición profesores y profesoras todo lo necesario.

Más concretamente: resulta absurdo hablar de que el profesorado se implique activamente en tareas de investigación e innovación (y con ello en la mejora de la enseñanza), mientras sigan existiendo currículos inabordables, escaso número de horas semanales para cada materia (que en muchos casos no son horas, sino periodos de 45 o 50 minutos), excesivo número de grupos a los que dar 
clase, bajos salarios, contenidos educativos que cambian cada vez que se da un cambio de gobierno, falta de medios e incentivos, exceso de horas lectivas totales, inestabilidad laboral, etc.

Pero, independientemente de esa situación (que requiere de una acción fundamentalmente política y sindical), los departamentos de didáctica de las ciencias de las universidades deberían esforzarse más por iniciar a los futuros profesores en la investigación didáctica, las revistas específicas por fomentar los trabajos de innovación y los investigadores en general por llevar a cabo, también, trabajos y proyectos a los que se incorpore el profesorado de aula.

\section{Aplicación mecánica de otros campos de conocimiento a la DCE}

La educación científica no está exenta aún de discusiones y polémicas acerca de qué enseñar y qué métodos de enseñanza conviene utilizar. En algunos países como España esas disputas se ven agravadas y fomentadas por unas políticas educativas erráticas y muy poco estables. Además, hay que tener en cuenta que la DCE, a pesar de los claros avances experimentados desde la década de 1980, aún no ha logrado ser percibida de forma generalizada como el cuerpo global y coherente de conocimientos que de hecho es. En sus inicios, era normal encontrar trabajos de investigación con una fundamentación teórica centrada en psicología educativa o pedagogía general. Afortunadamente, está situación ha cambiado y hoy ya es posible realizar la fundamentación teórica de los trabajos de investigación didáctica, utilizando el propio cuerpo de conocimientos de la DCE. No obstante, que sea posible no significa que sea algo general.

De Pro (2009) en relación a la brecha existente entre investigación y docencia, se sorprendía de la escasa participación del profesorado de secundaria en los últimos congresos de didáctica de las ciencias realizados en España, siendo que este colectivo fue un gran impulsor de las anteriores ediciones de dichos congresos, y señalaba la necesidad de investigar las causas de esa desconexión.

Sin duda, el problema es complejo y las causas varias (en este trabajo se han comentado ya algunas), pero quizás se pueda añadir el hecho de que bastantes profesores de secundaria piensan que una gran parte de los contenidos sobre los que se ocupa la DCE en la actualidad tienen una carga excesiva de Psicología, Pedagogía y Sociología, en donde continuamente se está cambiando el foco de atención (objetivos, capacidades, competencias...) y en donde (y esto es lo más grave) quedan relegados a un segundo plano los contenidos científicos propios de las materias a enseñar y los problemas concretos que conlleva el aprendizaje de los mismos, olvidando a menudo todo lo que ya se ha logrado o pretendiendo reinventarlo simplemente a base de un cambio en la terminología utilizada. Coincidimos plenamente, pues, con Micheleni et al 2013., cuando, refiriéndose a la didáctica de la Física, señalan que:

La formación inicial de los profesores es un campo en el que la investigación en la Didáctica de la Física ha trabajado durante mucho tiempo. No se debe confundir con la investigación pedagógica sobre la enseñanza o la investigación psicológica sobre el aprendizaje individual, o con los estudios sociológicos sobre la organización de la actividad escolar. Está vinculada a la construcción de competencias para producir aprendizaje específico de carácter disciplinar (el aprendizaje de la materia).

De acuerdo con lo expuesto, pensamos que sería conveniente que desde las facultades de educación y otros centros de formación del profesorado, se impulse la formación en contenidos específicos de DCE (Carrascosa et al., 2014), evitando que dicha formación quede reducida a conocimientos de Psicología y Pedagogía generales o que se contemple como una simple aplicación práctica de estos campos. 


\section{Incorporación escasa de nuevas necesi- dades formativas}

Desde hace tiempo, diversos organismos internacionales vienen Ilamando la atención para que los educadores contribuyamos a que la ciudadanía desarrolle un conocimiento adecuado sobre los graves problemas (contaminación, agotamiento de recursos, cambio climático, superpoblación...) a los que se enfrenta hoy la humanidad. Este Ilamamiento adquirió una gran relevancia en 1992, durante la Cumbre de la Tierra convocada por Naciones Unidas en Río de Janeiro. En dicha cumbre se pidió explícitamente que todos los educadores, cualquiera que fuese nuestro campo específico de trabajo, contribuyésemos a hacer posible la participación ciudadana en la búsqueda de soluciones a una situación de verdadera emergencia planetaria. Todo ello llevó a Naciones Unidas a la institución de una década de la educación por un futuro sostenible (2005-2014), en la que se pretendía lograr la implicación de todos los educadores y, posteriormente, a su continuación en el "Programa de acción global: un compromiso renovado por la educación para la sostenibilidad".

Cabe preguntarse, pues, cómo puede el profesorado de ciencias responder a esos Ilamamientos e incorporar más y mejor la problemática de la sostenibilidad a la educación (Vilches y Gil Pérez, 2013). Para ello es necesario superar algunas concepciones y comportamientos simplistas, como, por ejemplo, el hecho de que muchos docentes vean en ello (educación para la sostenibilidad), una "desviación" que puede perjudicar la preparación académica de sus alumnos, y que la problemática de la sostenibilidad impregne todo el currículum oficial incorporándose a los planes educativos con la importancia que merece.

Todo ello exige, además de leyes educativas apropiadas, que dicha problemática se contemple también en la formación inicial y permanente del profesorado $y$, consecuentemente, en la DCE, desde la que se han de desarrollar ejemplos y propuestas fundamentadas sobre las formas más eficientes de incorporar al currículo la educación para la sostenibilidad. Y ello, no solo en la didáctica específica de la Biología sino también en la del resto de didácticas específicas y en la DCE en general.

\section{Conclusiones}

La DCE se puede considerar ya como un cuerpo de conocimientos específicos, con un núcleo duro en el cual se encuentran, entre otros, aquellos resultados más directamente relacionados con aspectos que son claves para la enseñanza y aprendizaje de las ciencias (ideas alternativas, introducción de conceptos, resolución de problemas, trabajos prácticos, evaluación, etc.). En este trabajo se ha intentado mostrar que para que esos conocimientos puedan tener una incidencia mayor en la enseñanza y aumentar su percepción por parte del profesorado como una parte fundamental de un cuerpo específico de conocimientos, es necesario superar toda una serie de problemas que se oponen a ello.

La DCE que se imparte en las facultades de educación y en diferentes cursos y procesos de formación debería tenerlos muy en cuenta y tomar, en su caso, las decisiones oportunas para poder avanzar en su solución. Entre otras:

- Incorporar formación en algunos contenidos básicos, propios de la materia que los profesores han de enseñar y en la utilización didáctica de la Historia de la Ciencia y la Epistemología científica. Así como la formación en el uso adecuado de las nuevas TIC en la enseñanza y aprendizaje de las ciencias, evitando el absurdo de que las nuevas tecnologías vayan de la mano de viejas metodologías. Fomentar trabajos de investigación didáctica centrados en la elaboración de propuestas y ejemplos concretos sobre cómo utilizar applets, simuladores y otros recursos educativos digitales dentro de un modelo de enseñanza que se plantee de 
forma explícita desarrollar en los alumnos la competencia científica, con todo lo que ello implica.

- Llevar a cuestionar y transformar el pensamiento docente espontáneo, respecto a temas tales como: la evaluación; la naturaleza de la ciencia y el trabajo científico; o las relaciones entre ciencia, tecnología, sociedad y medio natural (CTSA).

- Impartir cursos extensos o intensivos de formación didáctica del profesorado en los que se estudien, al menos, los contenidos de DCE más relacionados con aspectos claves para la enseñanza y aprendizaje de las materias científicas (ideas alternativas, trabajos prácticos, resolución de problemas de lápiz y papel, relaciones CTSA, actitudes hacia la ciencia y su aprendizaje, evaluación, etc.). Y en donde sea posible percatarse no solo del interés de estos temas, sino también de la coherencia existente entre los distintos aspectos tratados en ellos, empleando unas estrategias metodológicas también coherentes con todo aquello que se propone.

- Fomentar la elaboración de materiales educativos concretos (temas o unidades didácticas) para desarrollar en el aula. En estos debe procurarse que se plasmen de forma coherente e integrada las principales orientaciones didácticas en aspectos claves como los citados, a título de ejemplo, en el párrafo anterior, incluyendo propuestas concretas para la evaluación del aprendizaje.

- Impulsar la introducción y familiarización de los profesores en formación en la investigación e innovación, planteando, por ejemplo, la réplica por su parte de investigaciones (o parte de investigaciones) ya realizadas, la elaboración de temas o unidades didácticas, la práctica en el aula de innovaciones educativas y análisis de sus resultados, etc.

- Centrar la investigación en problemas concretos de interés, relativos a la enseñanza y aprendizaje de los contenidos científicos presentes en el currículo. Evitar reducir la DCE a una mera aplicación de otros campos de conocimiento como la Psicología o Pedagogía generales.

- Favorecer la implicación del profesorado en tareas de investigación e innovación didáctica, a través de la realización de proyectos y trabajos de interés para la docencia en las aulas, prestando una atención particular a todo lo relacionado con la educación para la sostenibilidad.

El desarrollo e incorporación, en la medida que corresponda, de propuestas como las anteriores en los programas, cursos, materiales utilizados y actividades en general para la formación didáctica del profesorado de ciencias experimentales. Es algo necesario si queremos tener profesionales capaces de enseñar bien y hacerlo cada vez mejor.

Una continuación lógica del trabajo aquí presentado sería evaluar hasta qué punto se plantean y se llevan a cabo este tipo de propuestas en los distintos cursos, programas o másteres (maestrías) que se realizan para la formación del profesorado en diferentes universidades (públicas y privadas). Esto proporcionaría una base de datos importante sobre la situación actual y permitiría elaborar y adoptar, de forma fundamentada, medidas apropiadas con las que contribuir a una mejora realmente efectiva del proceso de formación didáctica del profesorado de ciencias en formación y en activo y, consecuentemente, de la enseñanza de las ciencias.

Por nuestra parte, hemos intentado mostrar aquí unas bases teóricas, acordes con la DCE, que podrían servir para guiar y fundamentar futuras evaluaciones, comentando algunos aspectos concretos cuya presencia (o ausencia) convendría estudiar y analizar de forma exhaustiva.

\section{Referencias}

Aragón-Méndez, M. M., García-Carmona, A. y Acevedo-Díaz, J. A. (2016). Aprendizaje de estudiantes de secundaria sobre la naturaleza de 
la ciencia mediante el caso histórico de Semmelweis y la fiebre puerperal. Revista Científica, 27, 302-317. Doi: 10.14483/udistrital.jour. $\underline{\text { RC.2016.27.a1 }}$

Alonso, M., Gil, D. y Martínez-Torregrosa, J. (1992). Concepciones espontáneas de los profesores de ciencias sobre la evaluación. Obstáculos a superar y propuestas de replanteamiento. Revista de Enseñanza de la Física, 5(2), 18-38.

Bañas, C., López, A., Mellado, V. y Ruiz, C. (2009). Metacognition and profession-al development of secondary education science teachers: a case study. Journal of Education Research, 3(12), 129-148.

Carrascosa, J. (2005). El problema de las concepciones alternativas en la actualidad (parte II). EI cambio de las concepciones alternativas. Revista Eureka sobre Enseñanza y Divulgación de las Ciencias, 2(3), 388-402.

Carrascosa, J., Domenech, J., Martínez, J., Osuna, L. y Verdú, R. (2014). Curso básico de Didáctica de las Ciencias. Enseñanza secundaria. Profesorado de ciencias en formación y en activo. Recuperado de: didacticafisicaquimica.es

Cortés Pascual, A., Cano Escoriaza, J. y Orejudo Hernández S. (2015). Competencias, valores laborales y formación previa antes y después del Practicum: un estudio con alumnado del Máster de Formación del Profesorado de Secundaria de la Universidad de Zaragoza. Investigación en la escuela, 85, 19-32.

De Pro Bueno, A. (2009). ¿Qué investigamos sobre la Didáctica de las Ciencias Experimentales en nuestro contexto educativo? Investigación en la escuela, 69, 45-69.

Fernández, I., Gil-Pérez, D., Valdés, P. y Vilches, A. (2005). ¿Qué visiones de la ciencia y la actividad científica tenemos y transmitimos? En: ¿Cómo promover el interés por la cultura científica? (capítulo 2, pp. 29-62). Santiago, Chile: Oreal, Unesco.

García-Carmona, A. y Acevedo, J. A. (2016). Concepciones de estudiantes de profesorado de educación primaria sobre naturaleza de la ciencia. Revista Mexicana de Investigación Educativa, 21(69), 583-610.

Gil, D., Carrascosa, J., Furió, C. y Martínez-Torregrosa, J. (1991). La enseñanza de las ciencias en la educación secundaria. Horsori. Barcelona.

Gil-Pérez, D. y Vilches, A. (2004). La formación del profesorado de ciencias de secundaria y de universidad. Educación Química, 15(1), 43-58.

Hewson, P. W. (2007). Teacher professional development in science. En: S. K. Abell y N. G. Lederman (eds.), Handbook of research on science education (pp. 1177-1203). Nueva Jersey: Lawrence Erlbaum Ass.

Kortaghen, F. (2010). La práctica, la teoría y la persona en la formación del profesorado. Revista Interuniversitaria de Formación del Profesorado, 68(24, 2), 83-101.

Marchesi, A., Martín, E., Casas, E., Ibáñez, A., Monguillot, I., Riviere, V. y Romero, F. (2005). Tecnología y aprendizaje. Investigación sobre el impacto del ordenador en el aula. Madrid: Ediciones SM.

Martín, C., Prieto, T. y Jiménez, A. (2015). Tendencias del profesorado de ciencias en formación inicial. Enseñanza de las Ciencias, 33(1), 167-184.

Michelini, M. (ed.) (2004). L'educazione Scientifica nel raccordo territorio/università a Udine. Udine: Forum.

Michelini, M., Santi, L. y Stefanel, A. (2013). La formación docente: un reto para la investigación. Revista Eureka sobre Enseñanza y Divulgación de las Ciencias, 10(número extraordinario), 846-870.

National Academies of Sciences, Engineering and Medicine. (2015). Science Teachers Learning: Enhancing Opportunities, Creating Supportive Contexts. National Academies Press.

Ritchie, S. M. (2008). Editorial: The next phase in scholarship and innovative research in science education. Research in Science Education, 38(1), 1-2. Doi: $\underline{10.1007 / s 11165-007-9071-8}$ 
Rocard, M., Csermely, P., Jorde, D., Lenzen, D., Walwerg-Henriksson, H. y Hemmo, V. (2007). Science Education Now: A Renewed Pedagogy for the Future of Europe. Bélgica: European Commission. Community Research. Recuperado de: http://ec.europa.eu/research/ science-society/document library/pdf 06/ report-rocard-on-science-education en.pdf

Russell, T. y Martin, A.K. (2007). Learning to teach science. Handbook of research on Science Education (pp. 1151-1178). Nueva York: Routledge, Taylor \& Francis group.

Sanmartí, N. e Izquierdo, M. (2001). Cambio y conservación en la enseñanza de las ciencias ante las TIC. Didáctica de las Ciencias Experimentales, 29, 71-83.

Solbes, J., Domínguez-Sales, M.C., Fernández-Sánchez, J., Furió, C., Cantó, J.R. y Guisasola, J. (2013). ¿El profesorado de Física y Química incorpora los resultados de la investigación en Didáctica? Didáctica de las Ciencias Experimentales y Sociales, 27, 155-178.

Solbes, J., Furió, C., Gavidia, V. y Vilches, A. (2004). Algunas consideraciones sobre la incidencia de la investigación educativa en la enseñanza de las ciencias. Investigación en la Escuela, 52, 103-110.

Solbes, J., Montserrat, R. y Furió, C. (2007). El desinterés del alumnado hacia el aprendizaje de la ciencia: implicaciones en su enseñanza. Didáctica de las Ciencias Experimentales y Sociales, 21, 91-117.

Taylor, P.C., Taylor, E. y Luitel, B.C. (2012). Multi-paradigmatic Transformative Research as/for Teacher Education: An Integral Perspective. En: B. J. Fraser, K. To-bin y McRobbie C. J., Second
International Handbook of Science Education (pp. 373-387). Dordrecht: Springer. Doi: $\underline{10.1007 / 978-1-4020-9041-7 \quad 26}$

Vázquez, A. y Manassero, M. A. (2008). El declive de las actitudes hacia la ciencia de los estudiantes: un indicador inquietante para la educación científica. Revista Eureka sobre Enseñanza y Divulgación de las ciencias, 5(3), 274-292.

Verdú, R., Martínez Torregrosa, J. y Osuna, L (2002). Enseñar y aprender en una estructura problematizada. Alambique, 34, 47.

Viennot, L., Chauvet, F. O., Colin, P. y Rebmann, G. (2005). Designing strategies and tools for teacher training: The role of critical details, examples in Optics. Science Education, 89(1), 13-27. Doi: $\underline{10.1002 / \text { sce.20040 }}$

Vilches, A y Gil Pérez, D. (2009). Una situación de emergencia planetaria a la que debemos y podemos hacer frente. Revista de Educación, número extraordinario, 101-122.

Vilches, A. y Gil Pérez, D. (2013). Ciencia de la sostenibilidad: un nuevo campo de conocimientos al que la Química y la Educación Química están contribuyendo. Educación Química, 24(2), 199-206. Doi: $\underline{10.1016 /}$ s0187-893x(13)72463-7

Waldrop, M. (2015). Why we are teaching science wrong and how to make it right. Nature, 523, 272- 274. Doi: $10.1038 / 523272 \mathrm{a}$

Wallace, J. y Loughran, J. (2012). Science Teacher Learning. En: B.J. Fraser, K. Tobin y McRobbie C. J., Second International Handbook of Science Education (pp. 295-306). Dordrecht: Springer. Doi: 10.1007/978-1-4020-9041-7 21 